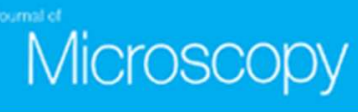

\title{
Making microscopy count: quantitative light microscopy of dynamic processes in living plants
}

\begin{tabular}{|r|l|}
\hline Journal: & Journal of Microscopy \\
\hline Manuscript ID & JMI-2015-0239.R1 \\
\hline Wiley - Manuscript type: & Themed Issue Paper \\
\hline Complete List of Authors: & $\begin{array}{l}\text { Fricker, Mark; University of oxford, Department of Plant Sciences } \\
\text { Moger, Julian ; University of Exeter, Physics } \\
\text { Littlejohn, George; University of Exeter, Biosciences } \\
\text { Deeks, Michael; University of Exeter, Biosciences }\end{array}$ \\
\hline Keywords: & Botanical microscopy, Bioimaging informatics, Live-cell imaging \\
\hline & \multicolumn{2}{|l}{} \\
\hline
\end{tabular}




\title{
Making microscopy count: quantitative light microscopy of dynamic processes in living plants.
}

\author{
Mark D. Fricker, Julian Moger, George R. Littlejohn, Michael J. Deeks
}

\begin{abstract}
Cell theory has officially reached 350 years of age as the first use of the word 'cell' in a biological context can be traced to a description of plant material by Robert Hooke in his historic publication "Micrographia: or some physiological definitions of minute bodies". The 2015 Royal Microscopical Society Botanical Microscopy meeting was a celebration of the streams of investigation initiated by Hooke to understand at the sub-cellular scale how plant cell function and form arises. Much of the work presented, and Honorary Fellowships awarded, reflected the advanced application of bioimaging informatics to extract quantitative data from micrographs that reveal dynamic molecular processes driving cell growth and physiology. The field has progressed from collecting many pixels in multiple modes to associating these measurements with objects or features that are meaningful biologically. The additional complexity involves object identification that draws on a different type of expertise from computer science and statistics that is often impenetrable to biologists. There are many useful tools and approaches being developed, but we now need more inter-disciplinary exchange to use them effectively. In this review we show how this quiet revolution has provided tools available to any personal computer user. We also discuss the oft-neglected issue of quantifying algorithm robustness and the exciting possibilities offered through the integration of physiological information generated by biosensors with object detection and tracking.
\end{abstract}

\section{Introduction}

Cell theory originated from early microscopical observations of plant material (Hooke 1665). The high contrast provided by the thickened cell walls of cork highlighted the precision of plant cell shape and the equally elegant higher-order arrangement of cells into tissue. These features were described and illustrated qualitatively in 1665 by Robert Hooke's Micrographia, but the mechanisms guiding plant cell development could only be hypothesised 300 years later. Upon reaching the $350^{\text {th }}$ anniversary of the publication of Micrographia the field of plant cell biology has entered a new era of (semi-)automated quantitative light microscopy. Previously qualitative forms of micrographical data are being efficiently mined with the aid of algorithms to determine rates and co-ordinates of change at the sub-cellular level. Dynamic processes operating at microsecond scales and distributed at sub-micron distances are responsible for driving the emergence of the features first noted by Hooke. However, for the most part these analysis techniques do not require novel microscopes, high-performance computing or deep knowledge of computer science. For the botanical quantitative evolution to gain pace plant scientists must be 
encouraged to use all of the analytical tools at their disposal. In this article we will explore some of these techniques, their implementation and their impact at the frontier of botanical microscopy. Our intention is to provide an overview of the computational approaches to data-extraction from dynamic micrographs so that plant biologists are aware of the powerful tools available and understand some of the core principles behind these methods. Increased knowledge of the workings of algorithms, combined with fluid communication between biologist and computer scientist, is needed to produce data of appropriate quality and practical algorithms for semiautomated analysis. Cross-disciplinary training opportunities, from undergraduate years onward, may act to encourage these interactions and strengthen the standards and practices of both research communities by entrenching mutual understanding. We hope that a deeper appreciation of these methods and their robust evaluation will further catalyse the use of quantitative botanical microscopy.

\section{Removing boundaries (and finding them again)}

The quantitative analysis of images is truly trans-disciplinary. Diverse academic fields share a need to deconstruct images into formats suitable for quantitative analysis. Nevertheless, structures within images and transitions between structures fall into a small number of feature categories that can be detected by algorithms and then used in higher-order processes to define objects and events (Smith \& Brady, 1997, Lowe, 2004, Bay et al., 2008, Maver, 2010). The mathematical theories underpinning many common feature-detection algorithms have a long history. For example, the 'watershed' algorithm has its origins in 1979 and was initially applied to micrographs of steel fractures by Beucher and Lantuejoul (Beucher \& Lantuéjoul, 1979). The digital imaging revolution and the developments predicted by Moore's Law of computing-power now allow its application to complex datasets without the need for access to specialist hardware. Its descendants are commonly used to segment biological structures into their component parts, such as organelles within a cell or cells within a tissue. From steel fractures, to bubbles, to cell walls, the members of the watershed family interpret all grey scale images as topographical landscapes in which the ridges define boundaries. Figure 1 demonstrates that the exact nature of the data is irrelevant. Both the copper-plate engravings of Hooke generated in 1665 and data from cutting-edge label-free fluorescence microscopy modalities can be segmented by software available to any PC user.

As the feature-set found in images is universal, the strategies for measuring these features and algorithms to automate such tasks are transferable between different contexts and even between different fields of science. For instance, the detection of 'blob-like' features within images can be exploited to track microscopic cellular organelles (Nelson et al., 2015) or, equally, suspicious human behaviour at railway stations (Elhamod \& Levine, 2013). It should be noted however that each algorithmic approach must be carefully optimised to new contexts. For instance, the boundaries in diffraction-limited fluorescence micrographs have distinct qualities compared to object boundaries in CCTV footage. Scientific imaging is progressively becoming 'object-orientated' in its methods. OBIA (Object Based Image Analysis) is a methodology developed during the last decade to analyse images of terrain generated by remote sensing techniques. This approach recognises and classifies objects into a defined hierarchy (reviewed by Blaschke, 2010). OBIA's key concept 
is to move beyond quantifying the spectral attributes of individual pixels to instead consider groupings of pixels that define objects; a revolution enabled by the affordable pixel-packing of competitive digital camera markets. Similar hierarchical strategies are being applied to micrographs of living multicellular organisms, such as the model nematode Caenorhabditis elegans to detect and quantify the attributes of specific organs and cells (Zhan et al., 2015). High-throughput digital biological imaging at all scales, from plantations to plastids, can benefit from automated feature detection and object-based quantitative analysis. Nevertheless, the challenge for plant scientists is how to navigate effectively from conceptually simple intensitybased segmentation techniques to exploit 'soft' or 'fuzzy' segmentation based on multiple characters with decision boundaries determined by supervised or unsupervised classifiers. Such approaches have been used extensively in highthroughput screening of animal cells growing in culture or even at the EM-level to assign organelles or cells to different classes using neural network classifiers (e.g. Boland and Murphy, 2001), k-means clustering (Coelho et al., 2013), support vector machines (SVM, Misselwitz et al., 2010) or cascaded hierarchical models (Perez et al., 2014). Whilst classifiers have been used in specific segmentation tasks in plants for nuclei (e.g. Schmidt et al., 2014, Skibbe et al., 2012) and for cells (Marcuzzo et al., 2009), it is only with incorporation of classification tools in free-to-download platforms such as CellProfiler (e.g. CellProfiler Analyst, Jones et al., 2008 or Enhanced CellClassifier, Misselwitz et al., 2010) that more plant microscopists may be tempted to try such approaches.

\section{Increasing Scale and Complexity}

Whilst a superb array of sophisticated microscope imaging platforms and fluorescent probes are now available for cell and sub-cellular imaging (Swanson et al., 2011, Shaw \& Ehrhardt, 2013, Fricker et al., 2006), what is especially exciting from a botanical perspective is the co-development of imaging techniques tailored to the challenges presented by macroscopic intact living plant tissues and scaling-up approaches to enable high-throughput phenotypic screening (e.g. Sozzani and Benfey, 2011). For example, displacement of air pockets from leaf spaces by airsaturated perfluorocarbons provides significant improvements in image quality for live-cell plant microscopy (Littlejohn et al., 2014), whilst new techniques, such as fluorescence intensity decay shape analysis microscopy (FIDSAM) may help to obviate issues with autofluorescence that plague plant imaging (Schleifenbaum et al., 2010). At an organ level, growing root systems can now be imaged in root arrays (Busch et al., 2012) or transparent soils (Downie et al., 2014, Downie et al., 2012) that allow long-term observation under minimal stress. This has in turn facilitated new optical approaches, such as light sheet tomography (Yang et al., 2013) which provides an extremely powerful approach for live-cell fluorescence microscopy to maximise fluorescence signal whilst minimising phototoxicity (Maizel et al., 2011) and can be combined with other modalities to quantify signalling and physiological dynamics across a developing root network (Costa et al., 2013). Alternatively, macroscopic imaging of intact plants in soil can be enhanced by constitutive expression of luciferase to aid root segmentation and analysis (GLO-roots, RellánÁlvarez et al., 2015), or by using other non-invasive imaging techniques, such as Xray tomography (Flavel et al., 2012, Mooney et al., 2012). Even relatively simple camera-based analysis of root architecture have now been extended to encompass 
high-throughput phenotyping platforms (Slovak et al., 2014, Galkovskyi et al., 2012, French et al., 2009).

\section{Generic software environments versus bespoke tools}

The increased number, size, dimensions and complexity of botanical images from multi-channel, super-resolution images at the nanometer scale, to phenotypic screens operating on 1000s of accessions, would benefit from parallel improvements in automated image analysis tools (Rousseau et al., 2015). An increasing number of bioimage informatics resources (summarised in figure 2) are available (Lobet et al., 2013), often through open, free-to-download platforms including ImageJ (Schneider et al., 2012), Fiji (Schindelin et al., 2012), CellProfiler (Kamentsky et al., 2011) and Icy (de Chaumont et al., 2012). In addition, bespoke software tools are available that integrate sub-cellular measurements of key parameters, such as cell geometry, nuclei volume and division state, in a tissue context defined by local co-ordinate systems that reflect the structure of the organ (French et al., 2009, Barbier de Reuille et al., 2015, de Reuille et al., 2014, Schmidt et al., 2014, Busch et al., 2012, Fernandez et al., 2010). These can be used to automatically build a complete 3D atlas of root structure (3DCellAtlas, Montenegro-Johnson et al., 2015, RootArray, Busch et al., 2012). Whilst the range of solutions emerging is admirable, there is also significant benefit in integration within existing frameworks to ensure extensibility and interoperability (Eliceiri et al., 2012). In addition, algorithm development and optimisation benefits from publically-accessible databases with test images and, ideally, ground truth data (Mairhofer et al., 2015). Thus, open source datasets such as the Plant Organelles Database (Mano et al., 2013) or the Live Images of Plant Stomata (LIPS) database (Higaki et al., 2013, Higaki et al., 2012) are just beginning to become available, but do not yet have ground truth data included.

The majority of cell-scale software development for bioimaging informatics has focussed on delineating cell boundaries, nuclear position and nuclear volume. For example, in root apices, Cell-Scale imaging (CellSeT, Pound et al., 2012) uses noise filtering, watershed segmentation, and active contours to refine the cell outlines from two-dimensional median root sections. Marcuzzo et al., (2009) also use watershed segmentation with region growing, but then extract a set of feature descriptors as input to a SVM to classify root cells. The iRoCS Toolbox (intrinsic root co-ordinate system, Schmidt et al., 2014) uses cylindrical co-ordinates with the origin at the quiescent centre (QC) and the main axes corresponding to the centreline of the root, the radial distance from the centreline, and the angle around the axis. Cells are identified by segmentation and additional features, such as the nuclei, are segmented using local 3D descriptors based on spherical harmonics that are then used to train a classifier (Schmidt et al., 2014, Skibbe et al., 2012). In shoot apices, multi-angle image acquisition, followed by three-dimensional reconstruction and cell segmentation with automated lineage tracking (MARS-ALT, Fernandez et al., 2010) or watershed-segmentation and local graph-matching (Liu et al., 2011) or tracking (Serrano-Mislata et al., 2015) can follow lineages in developing meristems.

Alternatively, whilst MorphoGraphX is specifically designed to capture dynamics of epidermal layers that are extracted from three-dimensional data sets of roots or shoots, whilst correctly retaining their three-dimensional curvature (Barbier de 
Reuille et al., 2015, Kierzkowski et al., 2012). This software is particularly well suited to analysing time series of cell growth as there is the option to co-segment multiple time points simultaneously, ensuring that segmented cell volumes are robustly paired across time. It is also possible to extract the growth anisotropy of each cell, quantifying the direction and amplitude of expansion. Quantification of cell anisotropy recalls the observation first described with Micrographia scheme xi:

"if you cut off a piece from a board of Cork transversly, to the flat of it, you will, as it were, split the pores, and they will appear just as they are express'd in the Figure B of the XI. Scheme. But if you shave off a very thin piece from this board, parallel to the plain of it, you will cut all the pores transversly, and they will appear almost as they are express'd in the Figure A"

Software solutions such as MorphoGraphX are being used to understand the actions driving and guiding this anisotropy at the tissue and cellular scale (Bassel et al., 2014). The attraction of these approaches is the ability to feed time-series data of cell size and shape into mathematical models that estimate the physical forces on the tissue (Hamant et al., 2008, Sampathkumar et al., 2014b, reviewed by Jensen \& Fozard, 2015, Sampathkumar et al., 2014a, Ali et al., 2014)

\section{Skewed Mass Action: Tissue-scale features emerge from quantifiable asymmetries in dynamic sub-cellular structures and events.}

Bioimaging informatic approaches are being applied at much finer scales of space and time to investigate the molecular mechanisms shaping cell anisotropy. The cell expansion model of Paul Green and his contemporaries postulated that the 'hooplike' orientation of cellulose microfibrils in the plant cell wall that had been observed using electron microscopy must constrain the plane of cell growth, thus dictating anisotropy (Green, 1962). It was deduced that the orientation of these cellulose microfibrils must in turn be controlled by signal-responsive tracks within the cell interior. In 2006 Ehrhardt and colleagues showed that cellulose synthase complexes (CesA) utilise microtubule arrays immediately beneath the plasma membrane as guides (Paredez et al., 2006). In this seminal paper the authors also showed that light acted as a stimulus to re-orientate microtubules and cellulose synthase trajectories (Paredez et al., 2006, Lindeboom, 2013). More recently physical stress and the directional flux of the plant hormone auxin have been demonstrated to guide microtubule orientation and (consequently) cell morphogenesis (Hamant et al., 2008, Heisler et al., 2010, Sampathkumar et al., 2014b, Sampathkumar et al., 2014a), whilst the actin cytoskeleton helps to regulate delivery and lifetime of plasmamembrane CesA complexes (Sampathkumar et al., 2013). The most recent of these high-impact studies utilises MorphographX (Barbier de Reuille et al., 2015). It is also relevant to note that quantification of the polar distribution of auxin efflux proteins is a feature within CellSeT, where the distribution of GFP fluorescence is plotted relative to the cell wall image to assign apical or basal distributions (Pound et al., 2012). The step-change achieved is the co-quantification of asymmetry in the distribution of specific molecules and the consequential development of asymmetries in cell morphology. The controlled pattern of growth emerges from a seemingly chaotic mass-action of molecular events such as the growth and shrinkage of 
microtubules. Key advances in our understanding of these mechanisms are achieved when particular processes are identified as being crucial in skewing organelle or molecular flux so that asymmetries are established. For example, the reorientation of microtubule arrays in response to physical pressure has been linked to an increase in microtubule severing frequency through the protein katanin (Sampathkumar et al., 2014a). Accurate quantification of fleeting molecular events can therefore be key to deducing mechanism.

\section{Sub-cellular object detection}

Whilst many plant publications still rely on visual inspection with limited quantitation using manually-defined regions-of-interest (ROIs), here we are interested in the problem of quantifying classes of object at the subcellular or even molecular scale using feature detection. Specific classes of object can be artificially highlighted by probes and stains (e.g. using a fluorescence labelling method) to produce extremely high-contrast images, or even imaged using label-free methods (see Box 1).

Fluorescent sub-cellular features can be reduced to three fundamental types (for review see Nelson et al., 2015): (1) Single molecules (Langhans et al., 2001); (2) 'Blobs', defined as regions of shared image brightness that constitute an object; (3) Curvilinear / filamentous structures, such as the multiple networks contained within the eukaryote cytoplasm, including the endoplasmic reticulum and cytoskeletal arrays (for review see Nelson et al., 2015).

In the case of blob-like organelles, segmentation approaches have progressed from simple intensity-thresholding, through adaptive thresholding, pattern-matching templates to multi-feature classifiers (Nelson et al., 2015). The advantage of objectdetection over pixel-based classifiers for co-localisation studies (e.g. JACoP plugin for ImageJ, Bolte and Cordelieres, 2006), is a quantitative estimate of the distribution of distances between objects, rather than single statistical measure from the whole image. Distance metrics are also more relevant to analysis of spatial associations, such as organelles travelling on the cytoskeleton, than co-localisation indices (Nelson et al., 2015).

In the case of filamentous structures such as the microtubule cytoskeleton, the ends of the microtubules are initially detected using microtubule plus-end binding proteins modelled as anisotropic Gaussians. Particle tracking is usually split into two steps; object detection and object linking. The optimal strategy for linking varies depending on the data (Smal \& Meijering, 2015). The single particles that are detected in each frame are then linked to form a track segment based on current speed and direction, but using an algorithm that minimises the global cost of all connections made (Jaqaman et al., 2008). The track segments linked to form a trajectory using the same approach. Whilst plus-end proteins only reveal growing microtubules, it is possible to infer paused or shrinking microtubules from the same data if these events are collinear and separated by a short time interval (Applegate et al., 2011, Jaqaman et al., 2008, Matov et al., 2010).

Less attention has focussed on analysis of the cytoskeletal network structure itself. The overall pattern of organisation can be inferred from macroscopic properties such as the texture, measured using empirical mode decomposition (Lu et al., 2014), 
nematic tensors using FibrilTool (Boudaoud et al., 2014), which is based on the first derivative of the gradient, or maximum projection analysis using a virtual polariser, (MicrofilamentAnalyser (MFA) Jacques et al., 2013). The actin cytoskeleton in root hairs has been characterised by mathematical morphology techniques (Kimori et al., 2016) and microtubule orientation has been identified using anisotropic intensity kernels applied over a range of angles (implemented as the Loco plugin for imageJ (Lindeboom et al., 2013). Sampathkumar (Sampathkumar et al., 2014a) used a nematic tensor based tool to characterise microtubule orientations in parallel with AFM measurement of wall mechanical properties and finite-element modelling to predict patterns of wall stress. They were also able to determine feedback between tissue stress and microtubule re-alignment using imposed pressure, cell ablation, pharmacological agents and katanin severing mutants (Sampathkumar et al., 2014a)

Identification and segmentation of the network itself is more challenging. Most algorithms in network analysis were developed for tracing vasculature systems or neurons and typically include an image enhancement step that emphasises the 'ridge' nature of the structure. The most common approaches use a second-order derivatives of a Gaussian over a range of angles and scales (e.g. Frangi et al., 1998), although pattern matching templates can also be used (Basu et al., 2015).

Anisotropic kernels are more powerful as they emphasise the elongated shape of network segments (Lopez-Molina et al., 2015, Meijering et al., 2004). The maximum response of the filter provides information on the edge strength and its orientation, whilst additional measures, such as the anisotropy of the first three eigenvalues, gives a measure of the edge 'vesselness' (Frangi et al., 1998) or 'neuriteness' (Meijering et al., 2004). An alternative approach uses local phase-congruency to give an intensity-independent approach to edge enhancement, which is critical when the edges vary in intensity as well as scale and orientation. The phase-congruency measure can also be combined with tensor-based measurements to give phasecongruency 'vesselness' or 'neuriteness' (Obara et al., 2012b, Obara et al., 2012a)

The enhancement step is followed by segmentation using non-maximal suppression and hysteresis thresholding (Lopez-Molina et al., 2015), a watershed algorithm and edge pruning (Obara et al., 2012b) or a reverse diffusion-based algorithm (Basu et al., 2015) to give a pixel-based skeleton. The resulting image skeleton is then converted to a graph representation, where each junction is classed as a node and the intervening segment a link. Whilst these approaches provide a wealth of graphtheoretic measures to help characterise the network, they also automatically allocate a node to every filament crossing, irrespective of whether there is a real biological interaction of the cytoskeletal elements at each point. Disentangling overlapping filaments is both theoretically and computationally challenging, nevertheless robust, automated solutions have been developed recently and tested on plant actin networks (DeFiNe, Breuer and Nikolowski, 2015).

Alternative approaches do not attempt to accurately segment the individual filament, but impose a regular sampling lattice and estimate the strength of the connections on that lattice from the intensity of the pixels intersecting the edges (Breuer et al., 2014). This is a relatively straightforward approach that can detect differences in overall behaviour in the network structure and dynamics between treatments, albeit with an approximation representation of the actual network (Breuer et al., 2014). 


\section{Who Watches the Watchers}

Segmentation of complex sub-cellular structures is challenging. Ideally the performance of each method should be evaluated against a set of ground truth images that are representative of the range of features to be analysed, including levels of noise (e.g. Lopez-Molina et al., 2013, Perez et al., 2013, Mairhofer et al., 2015). Typically the ground truth has to be defined by a human observer (or ideally cross-referenced between multiple observers), as the human visual processing system still represents the gold-standard in pattern recognition. Performance is measured in terms of correctly identified pixels or objects (true positives, TPs or hits), spurious responses (false positives, FPs), true negatives (TNs), and false negatives (FNs or misses). The results are summarised by various metrics or Error Measures (EMs) (Lopez-Molina et al., 2013) such as Receiver Operating Characteristic (ROC) or Precision-Recall (P-R) graphs. Whilst a single measure can describe the performance of the algorithm, it is helpful to determine the optimal level of the threshold used to distinguish feature from background from a complete ROC or P-R curve.

The hit or miss classification itself is a rather blunt instrument, as it does not capture the extent that the pixel classification is in error, so for example a single pixel shift arising from a slight displacement in detection of the ridge centreline in a skeleton image from the cytoskeleton might generate an almost complete mismatch between the skeleton and the ground-truth, when it could actually be a remarkably good analysis with a small offset. One approach is to allow greater tolerance in the hit-ormiss decision (Obara et al., 2012b, Obara et al., 2012a). Alternatively distancebased EMs include a quantitative distance metric of how far apart the distributions are, such as Baddeleys Delta Metric (BDM) (Lopez-Molina et al., 2013).

Whilst there is a long history of validation for tracking and segmentation algorithms in computer vision, similar testing against ground-truth images is not yet common place for other types of automated image analysis in plant sciences and is only just beginning to emerge in biomedical domain (Ljosa et al., 2012). One clear need for the community is to develop a suitable collection of datasets available alongside the analysis algorithms with ground truth validation.

\section{A fifth dimension - Quantitative physiological imaging with genetically encoded sensors}

Spectral and signal intensity information from fluorescent probes can be used to measure the chemical environment within or around an identified object. By using genetically encoded sensors it is possible to combine the powers of minimallyinvasive object-orientated live cell imaging with sub-cellular physiological measurements. This approach to live-cell imaging adds a new dimension to the data that can be extracted from micrographs and has been used with increasing sophistication in botanical microscopy to report on the physiological status of whole 
cells and individual organelles. These techniques generally involve direct binding of the chemical of interest, which in turn may lead to an increase or decrease (e.g. the auxin reporter DIl-venus (Brunoud et al., 2012)) in signal intensity of the biosensor, using a single excitation wavelength and single emission. Alternatively, biosensors may involve ratiometric sensing, where dual excitation, single emission (e.g. HyPer Beluosov et al., 2006, Costa et al., 2010) or single excitation, dual emission (e.g. CaMeleons Miyawaki et al.,1997, Allen et al., 1999) generates two signal outputs, which vary in their relative intensities as the concentration of the species being sensed varies. These two signals may then be used to give a ratiometric measure of species of interest concentration. The latest generation of transgenic single excitation, dual emission sensors employ Forster Resonance Energy Transfer (FRET) to cause excitation of one fluorophore by another, which is conditional both fluorophores of the FRET pair being in close enough proximity for FRET to occur. This mechanism for biosensing normally relies on a conformational change of the sensor on binding the moiety of interest bringing the interacting fluorophores together. The advantage of ratiometric sensors is that their ability to report the calibrated concentration of a chemical species of interest is independent of their own concentration. A wide range of genetically-encoded probes have been developed to quantitatively report concentration of a range of moieties including metabolites such as sucrose and glucose (Chaudhuri et al., 2008), the plant hormones jasmonic acid (Larrieu et al. 2015), auxin (Brunoud et al, 2012), and ABA (Waadt et al., 2014, Jones et al., 2014), and signalling components, most notably $\mathrm{Ca}^{2+}$ (Allen et al., 1999), ROS (HyPer (Costa et al., 2010) and redox potential (roGFP, Meyer \& Dick, 2010, Schwarzlander et al., 2008, Fricker, 2015, Schwarzländer et al., 2015). These have mostly been developed in non-plant systems and later implemented in plants, but recent years has seen the development of biosensors for jasmonic acid, auxin, ABA and sucrose, which have been designed to co-opt endogenous plant mechanisms for detecting these moieties. Plant scientists are also showing the way on the robust and thorough characterisation of genetically encoded biosensors. The recent work of Schwarzlander et al., (2014) has shown that pH sensitivity of some biosensors can compromise their utility and that users of these molecular tools must be alert to this problem.

The kind of molecular eavesdropping that genetically encoded biosensors provide is minimally invasive and, when combined with image segmentation techniques explored in this article, may yield great insights into physiological and signalling processes in plants. Furthermore, transgenic reporters can be targeted to different organelles allowing physiological measurements for distinct compartments when combined with object-based segmentation (Schwarzländer et al., 2012, 2014, Fricker, 2015, Fuchs et al., 2016) to reveal highly spatially localised, dynamic behaviour that cannot be capture by metabolomics or genomic approaches such as oscillations in nuclear calcium during rhizobium infection (Sun et al., 2007, Capoen, et al., 2011), pulsing of ROS and pH in mitochondria (Schwarzländer et al., 2012, 2014), redox regulation of plastid stromules (Brunkard et al. 2015) or highly localised mitochondrial redox changes during pathogen infection (Fuchs et al., 2016). 


\section{Conclusion}

Botanical microscopy has entered an object-based era of quantitative imaging supported by a wide range of software solutions and packages for programming languages (figure 2). These are often developed for specific tasks but can be applied with minimal modification to a wide range of scales and modalities. An increasing awareness of image analysis toolboxes within programming environments such as Python, Octave and Matlab has encouraged many biologists to learn how to perform basic modifications to scripts in order to adapt algorithms to novel datasets. Moreover, new probes and methods such as label-free imaging (Figure 1., Box 1.) are increasing the depth of information contained within live-cell imaging data of plant cells. These techniques will continue to develop in their sensitivity, range of targets and non-invasive properties. Cross-disciplinary interaction must be embraced to ensure that new opportunities for hypothesis testing through quantitative imaging data are not missed and that the algorithms applied are sufficiently robust.

\section{References}

Ali, O., Mirabet, V., Godin, C. \& Traas, J. (2014) Physical models of plant development. Annu. Rev. Cell Dev. Biol., 30, 59-78.

Allen, G. J., Kwak, J. M., Chu, S. P., Llopis, J., Tsien, R. Y., Harper, J. F., \& Schroeder, J. I. (1999). Cameleon calcium indicator reports cytoplasmic calcium dynamics in Arabidopsis guard cells. The Plant Journal, 19(6), 735747.

Applegate, K. T., Besson, S., Matov, A., Bagonis, M. H., Jaqaman, K. \& Danuser, G. (2011) plusTipTracker: Quantitative image analysis software for the measurement of microtubule dynamics. J. Struct. Biol., 176, 168-184.

Barbier de Reuille, P., Routier-Kierzkowska, A.-L., Kierzkowski, D., Bassel, G. W., Schüpbach, T., Tauriello, G., Bajpai, N., Strauss, S., Weber, A., Kiss, A., Burian, A., Hofhuis, H., Sapala, A., Lipowczan, M., Heimlicher, M. B., Robinson, S., Bayer, E. M., Basler, K., Koumoutsakos, P., Roeder, A. H. K., Aegerter-Wilmsen, T., Nakayama, N., Tsiantis, M., Hay, A., Kwiatkowska, D., Xenarios, I., Kuhlemeier, C. \& Smith, R. S. (2015) MorphoGraphX: A platform for quantifying morphogenesis in 4D. eLife, 4.

Bassel, G. W., Stamm, P., Mosca, G., Barbier de Reuille, P., Gibbs, D. J., Winter, R., Janka, A., Holdsworth, M. J. \& Smith, R. S. (2014) Mechanical constraints imposed by $3 \mathrm{D}$ cellular geometry and arrangement modulate growth patterns in the Arabidopsis embryo. PNAS USA, 111, 8685-8690.

Basu, S., Liu, C. \& Rohde, G. K. (2015) Localizing and extracting filament distributions from microscopy images. J. Microsc., 258, 13-23.

Bay, H., Ess, A., Tuytelaars, T. \& Van Gool, L. (2008) Speeded-up robust features (SURF). Computer vision and image understanding, 110, 346-359.

Belousov, V. V., Fradkov, A. F., Lukyanov, K. A., Staroverov, D. B., Shakhbazov, K. S., Terskikh, A. V., \& Lukyanov, S. (2006). Genetically encoded fluorescent indicator for intracellular hydrogen peroxide. Nature Methods, 3(4), 281-286.

Beucher, C Lantuéjoul. Use of watersheds in contour detection. International Workshop on image processing, real-time edge and motion detection/estimation, Rennes, France, Sept. 1979. 
Blaschke, T. (2010) Object based image analysis for remote sensing. ISPRS journal of photogrammetry and remote sensing, 65, 2-16.

Boland, M. V. \& Murphy, R. F. (2001) A neural network classifier capable of recognizing the patterns of all major subcellular structures in fluorescence microscope images of HeLa cells. Bioinformatics, 17, 1213-1223.

Bolte, S and Cordelières, F. P. (2008) A guided tour into subcellular colocalization analysis in light microscopy, J. Microsc. 224, 213-232.

Boudaoud, A., Burian, A., Borowska-Wykręt, D., Uyttewaal, M., Wrzalik, R., Kwiatkowska, D. \& Hamant, O. (2014) FibrilTool, an ImageJ plug-in to quantify fibrillar structures in raw microscopy images. Nat. Protocols, 9, 457463.

Breuer, D., Ivakov, A., Sampathkumar, A., Hollandt, F., Persson, S. \& Nikoloski, Z. (2014) Quantitative analyses of the plant cytoskeleton reveal underlying organizational principles. J. Roy. Soc. Interface, 11.

Breuer, D. \& Nikoloski, Z. (2015) DeFiNe: an optimisation-based method for robust disentangling of filamentous networks. Scientific Reports, $\mathbf{5}$.

Brunoud, G., Wells, D.M., Oliva, M., Larrieu, A., Mirabet, V., Burrow, A.H., Beeckman, T., Kepinski, S., Traas, J., Bennett, M.J. and Vernoux, T. (2012). A novel sensor to map auxin response and distribution at high spatio-temporal resolution. Nature, 482, 103-106.

Brunkard, J. O., Runkel, A. M. \& Zambryski, P. C. (2015). Chloroplasts extend stromules independently and in response to internal redox signals. PNAS USA, 112, 10044-10049.

Busch, W., Moore, B. T., Martsberger, B., Mace, D. L., Twigg, R. W., Jung, J., Pruteanu-Malinici, I., Kennedy, S. J., Fricke, G. K., Clark, R. L., Ohler, U. \& Benfey, P. N. (2012) A microfluidic device and computational platform for high-throughput live imaging of gene expression. Nat Meth, 9, 1101-1106.

Capoen, W., Sun, J., Wysham, D., Otegui, M. S., Venkateshwaran, M., Hirsch, S., Miwa, H., Downie, J. A., Morris, R. J., Ané, J.-M. \& Oldroyd, G. E. D. (2011) Nuclear membranes control symbiotic calcium signaling of legumes. PNAS USA, 108, 14348-14353.

Chaudhuri B., Hormann F., Lalonde S., Brady S.M., Orlando D.A., Benfey P., Frommer W.B., Protonophore- and $\mathrm{pH}$-insensitive glucose and sucrose accumulation detected by FRET nanosensors in Arabidopsis root tips. (2008). Protonophore- and $\mathrm{pH}$-insensitive glucose and sucrose accumulation detected by FRET nanosensors in Arabidopsis root tips., 56(6), 948-962.

Coelho, L. P., Kangas, J. D., Naik, A. W., Osuna-Highley, E., Glory-Afshar, E., Fuhrman, M., Simha, R., Berget, P. B., Jarvik, J. W. \& Murphy, R. F. (2013) Determining the subcellular location of new proteins from microscope images using local features. Bioinformatics, 29, 2343-2349.

Costa, A., Candeo, A., Fieramonti, L., Valentini, G. \& Bassi, A. (2013) Calcium dynamics in root cells of arabidopsis thaliana visualized with selective plane illumination microscopy. PLoS ONE, 8, e75646.

Costa, A., Drago, I., Behera, S., Zottini, M., Pizzo, P., Schroeder, J. I., et al. (2010). $\mathrm{H}_{2} \mathrm{O}_{2}$ in plant peroxisomes: an in vivo analysis uncovers a $\mathrm{Ca}^{2+}$-dependent scavenging system. The Plant Journal, 62(5), 760-772.

de Chaumont, F., Dallongeville, S., Chenouard, N., Herve, N., Pop, S., Provoost, T., Meas-Yedid, V., Pankajakshan, P., Lecomte, T., Le Montagner, Y., Lagache, T., Dufour, A. \& Olivo-Marin, J.-C. (2012) Icy: an open bioimage informatics platform for extended reproducible research. Nat Meth, 9, 690-696. 
de Reuille, P., Robinson, S. \& Smith, R. (2014) Quantifying Cell Shape and Gene Expression in the Shoot Apical Meristem Using MorphoGraphX. In: Plant Cell Morphogenesis (eds. V. Žárský \& F. Cvrčková). Humana Press.

Downie, H., Holden, N., Otten, W., Spiers, A. J., Valentine, T. A. \& Dupuy, L. X. (2012) Transparent soil for imaging the rhizosphere. PLOS ONE, 7, e44276.

Downie, H. F., Valentine, T. A., Otten, W., Spiers, A. J. \& Dupuy, L. X. (2014) Transparent soil microcosms allow 3D spatial quantification of soil microbiological processes in vivo. Plant Signaling \& Behavior, 9, e970421.

Elhamod, M. \& Levine, M. D. (2013) Automated real-time detection of potentially suspicious behavior in public transport areas. IEEE Transactions on Intelligent Transportation Systems, 14, 688-699.

Eliceiri, K. W., Berthold, M. R., Goldberg, I. G., Ibanez, L., Manjunath, B. S., Martone, M. E., Murphy, R. F., Peng, H., Plant, A. L., Roysam, B., Stuurman, N., Swedlow, J. R., Tomancak, P. \& Carpenter, A. E. (2012) Biological imaging software tools. Nat Meth, 9, 697-710.

El-Mashtoly, S. F., Niedieker, D., Petersen, D., Krauss, S. D., Freier, E., Maghnouj, A., Mosig, A., Hahn, S., Kotting, C. \& Gerwertt, K. (2014) Automated Identification of Subcellular Organelles by Coherent Anti-Stokes Raman Scattering. Biophys. J., 106, 1910-1920.

Fernandez, R., Das, P., Mirabet, V., Moscardi, E., Traas, J., Verdeil, J.-L., Malandain, G. \& Godin, C. (2010) Imaging plant growth in 4D: robust tissue reconstruction and lineaging at cell resolution. Nat Meth, 7, 547-553.

Flavel, R. J., Guppy, C. N., Tighe, M., Watt, M., McNeill, A. \& Young, I. M. (2012) Non-destructive quantification of cereal roots in soil using high-resolution $\mathrm{X}$ ray tomography. J. Exp. Bot. 63, 2503-2511

Frangi, A., Niessen, W., Vincken, K. \& Viergever, M. (1998) Multiscale vessel enhancement filtering. In: Medical Image Computing and Computer-Assisted Interventation (eds. W. Wells, A. Colchester \& S. Delp). Springer Berlin / Heidelberg.

French, A., Ubeda-Tomás, S., Holman, T. J., Bennett, M. J. \& Pridmore, T. (2009) High-throughput quantification of root growth using a novel image-analysis tool. Plant Physiol., 150, 1784-1795.

Fricker, M., Runions, J. \& Moore, I. (2006) Quantitative fluorescence microscopy: From art to science. Annu. Rev. Plant Biol., 57, 79-107.

Fricker, M. D. (2015) Quantitative Redox Imaging Software. Antioxidants \& Redox Signaling doi:10.1089/ars.2015.6390

Fuchs, R., Kopischke, M., Klapprodt, C., Hause, G., Meyer, A. J., Schwarzländer, M., Fricker, M. D. \& Lipka, V. (2015) Immobilized subpopulations of leaf epidermal mitochondria mediate PEN2-dependent pathogen entry control in Arabidopsis. The Plant Cell. doi:10.1105/tpc.15.00887

Galkovskyi, T., Mileyko, Y., Bucksch, A., Moore, B., Symonova, O., Price, C., Topp, C., lyer-Pascuzzi, A., Zurek, P., Fang, S., Harer, J., Benfey, P. \& Weitz, J. (2012) GiA Roots: software for the high throughput analysis of plant root system architecture. BMC Plant Biol., 12, 1-12.

Green, P. B. (1962) Mechanism for plant cellular morphogenesis. Science, 138, 1404-1405.

Hamant, O., Heisler, M. G., Jonsson, H., Krupinski, P., Uyttewaal, M., Bokov, P., Corson, F., Sahlin, P., Boudaoud, A., Meyerowitz, E. M., Couder, Y. \& Traas, J. (2008) Developmental Patterning by Mechanical Signals in Arabidopsis. Science, 322, 1650-1655. 
Heisler, M. G., Hamant, O., Krupinski, P., Uyttewaal, M., Ohno, C., Jönsson, H., Traas, J., Meyerowitz, E. M., Goldfarb, T. \& Lichten, M. (2010) Alignment between PIN1 polarity and microtubule orientation in the shoot apical meristem reveals a tight coupling between morphogenesis and auxin transport. PLoS biology, 8, 2409.

Higaki, T., Kutsuna, N. \& Hasezawa, S. (2013) LIPS database with LIPService: a microscopic image database of intracellular structures in Arabidopsis guard cells. BMC Plant Biol., 13, 81.

Higaki, T., Kutsuna, N., Hosokawa, Y., Akita, K., Ebine, K., Ueda, T., Kondo, N. \& Hasezawa, S. (2012) Statistical organelle dissection of Arabidopsis guard cells using image database LIPS. Scientific Reports, 2, 405.

Hooke R. Micrographia or some physiological descriptions of minute bodies made by magnifying glasses with observations and inquiries thereupon. London: Royal Society; 1665.

Jacques, E., Buytaert, J., Wells, D. M., Lewandowski, M., Bennett, M. J., Dirckx, J., Verbelen, J. P. \& Vissenberg, K. (2013) MicroFilament Analyzer, an image analysis tool for quantifying fibrillar orientation, reveals changes in microtubule organization during gravitropism. Plant J., 74, 1045-1058.

Jaqaman, K., Loerke, D., Mettlen, M., Kuwata, H., Grinstein, S., Schmid, S. L. \& Danuser, G. (2008) Robust single-particle tracking in live-cell time-lapse sequences. Nat. Methods, 5, 695-702.

Jensen, O. E. \& Fozard, J. A. (2015) Multiscale Models in the Biomechanics of Plant Growth. Physiology, 30, 159-166.

Jones AM, Danielson JÅH, ManojKumar SN, Lanquar V, Grossman G, Frommer WB. 2014. Abscisic acid dynamics in roots detected with genetically encoded FRET sensors. eLife 3:e01741. doi: 10.7554/eLife.01741

Kamentsky, L., Jones, T. R., Fraser, A., Bray, M.-A., Logan, D. J., Madden, K. L., Ljosa, V., Rueden, C., Eliceiri, K. W. \& Carpenter, A. E. (2011) Improved structure, function and compatibility for CellProfiler: modular high-throughput image analysis software. Bioinformatics, 27, 1179-1180.

Kierzkowski, D., Nakayama, N., Routier-Kierzkowska, A.-L., Weber, A., Bayer, E., Schorderet, M., Reinhardt, D., Kuhlemeier, C. \& Smith, R. S. (2012) Elastic domains regulate growth and organogenesis in the plant shoot apical meristem. Science, 335, 1096-1099.

Kimori, Y., Hikino, K., Nishimura, M. \& Mano, S. (2016) Quantifying morphological features of actin cytoskeletal filaments in plant cells based on mathematical morphology. J. Theor. Biol., 389, 123-131.

Langhans, M., Ratajczak, R., Lutzelschwab, M., Michalke, W., Wachter, R., FischerSchliebs, E. \& Ullrich, C. I. (2001) Immunolocalization of plasma-membrane $\mathrm{H}^{+}$-ATPase and tonoplast-type pyrophosphatase in the plasma membrane of the sieve element-companion cell complex in the stem of Ricinus communis $L$. Planta, 213, 11-19.

Larrieu A., Champion A., Legrand J., Lavenus J., Mast D., Brunoud G., Oh J., Guyomarc'h S., Pizot M, Farmer E.E., Turnbull C., Vernoux T., Bennett M.J., and Laplaze L. (2015). A fluorescent hormone biosensor reveals the dynamics of jasmonate signalling in plants. Nature Communications, 6, 6043.

Lindeboom, J. J., Nakamura, M., Hibbel, A., Shundyak, K., Gutierrez, R., Ketelaar, T., Emons, A. M. C., Mulder, B. M., Kirik, V. \& Ehrhardt, D. W. (2013) A mechanism for reorientation of cortical microtubule arrays driven by microtubule severing. Science, 342. Page numbers 
Littlejohn, G. R., Mansfield, J. C., Christmas, J. T., Witterick, E., Fricker, M. D., Grant, M. R., Smirnoff, N., Everson, R. M., Moger, J. \& Love, J. (2014) An update: improvements in imaging perfluorocarbon-mounted plant leaves with implications for studies of plant pathology, physiology, development and cell biology. Front Plant Sci, 5, 140.

Littlejohn, G. R., Mansfield, J. C., Parker, D., Lind, R., Perfect, S., Seymour, M., et al. (2015). In vivo chemical and structural analysis of plant cuticular waxes using stimulated raman scattering microscopy. Plant Physiology, May 2015, Vol. 168, pp. 18-28

Liu, M., Chakraborty, A., Singh, D., Yadav, R. K., Meenakshisundaram, G., Reddy, G. V. \& Roy-Chowdhury, A. (2011) Adaptive cell segmentation and tracking for volumetric confocal microscopy images of a developing plant meristem. Mol. Plant, 4, 922-931.

Ljosa, V., Sokolnicki, K. L. \& Carpenter, A. E. (2012) Annotated high-throughput microscopy image sets for validation. Nat Meth, 9, 637-637.

Lobet, G., Draye, X. \& Périlleux, C. (2013) An online database for plant image analysis software tools. Plant Methods, 9, 1-8.

Lopez-Molina, C., De Baets, B. \& Bustince, H. (2013) Quantitative error measures for edge detection. Pattern Recognition, 46, 1125-1139.

Lopez-Molina, C., Vidal-Diez de Ulzurrun, G., Baetens, J. M., Van den Bulcke, J. \& De Baets, B. (2015) Unsupervised ridge detection using second order anisotropic Gaussian kernels. Signal Processing, 116, 55-67.

Lowe, D. G. (2004) Distinctive image features from scale-invariant keypoints. Int.J Comp. Vis., 60, 91-110.

Lu, Y., Huang, C., Wang, J. \& Shang, P. (2014) An improved quantitative analysis method for plant cortical microtubules. In: TheScientificWorldJournal. 10, 2014:637183.

Mairhofer, S., Sturrock, C., Wells, D. M., Bennett, M. J., Mooney, S. J. \& Pridmore, T. P. (2015) On the evaluation of methods for the recovery of plant root systems from X-ray computed tomography images. Functional Plant Biology, 42, 460470.

Maizel, A., von Wangenheim, D., Federici, F., Haseloff, J. \& Stelzer, E. H. (2011) High-resolution live imaging of plant growth in near physiological bright conditions using light sheet fluorescence microscopy. Plant J., 68, 377-385.

Maker PD and Terhune R.W.,(1965) Study of optical effects due to an induced polarization third order in the electric field strength Phys. Rev. 137 A801

Mano, S., Nakamura, T., Kondo, M., Miwa, T., Nishikawa, S.-i., Mimura, T., Nagatani, A. \& Nishimura, M. (2013) The plant organelles database 3 (podb3) update 2014: integrating electron micrographs and new options for plant organelle research. Plant Cell Physiol. 55:e1. doi: 10.1093/pcp/pct140

Mansfield, J. C., Littlejohn, G. R., Seymour, M. P., Lind, R. J., Perfect, S., \& Moger, J. (2013). Label-free chemically specific imaging in planta with stimulated Raman scattering microscopy. Analytical Chemistry, 85(10), 5055-5063.

Marcuzzo, M., Quelhas, P., Campilho, A., Mendonca, A. M. \& Campilho, A. (2009) Automated Arabidopsis plant root cell segmentation based on SVM classification and region merging. Comp. Biol. Med., 39, 785-793.

Matov, A., Applegate, K., Kumar, P., Thoma, C., Krek, W., Danuser, G. \& Wittmann, T. (2010) Analysis of microtubule dynamic instability using a plus-end growth marker. Nat. Methods, 7, 761-U134. 
Maver, J. (2010) Self-similarity and points of interest. IEEE Trans. Pattern Analysis Machine Int., 32, 1211-1226.

Meijering, E., Jacob, M., Sarria, J. C. F., Steiner, P., Hirling, H. \& Unser, M. (2004) Design and validation of a tool for neurite tracing and analysis in fluorescence microscopy images. Cytometry, 58A, 167-176.

Meyer, A. J. \& Dick, T. P. (2010) Fluorescent protein-based redox probes. Antioxid Redox Signal, 13, 621-650.

Min W, Freudiger CW, Lu S, Xie XS. (2011) Coherent nonlinear optical imaging: beyond fluorescence microscopy. Annu Rev Phys Chem. 62, 507-30.

Misselwitz, B., Strittmatter, G., Periaswamy, B., Schlumberger, M. C., Rout, S., Horvath, P., Kozak, K. \& Hardt, W. D. (2010) Enhanced CellClassifier: a multiclass classification tool for microscopy images. BMC Bioinf., 11.

Miyawaki A, Llopis J, Heim R, McCaffery JM, Adams JA, Ikura M, Tsien RY. 1997. Fluorescent indicators for $\mathrm{Ca}^{2+}$ based on green fluorescent proteins and calmodulin. Nature 388, 882-887.

Montenegro-Johnson, T. D., Stamm, P., Strauss, S., Topham, A. T., Tsagris, M., Wood, A. T. A., Smith, R. S. \& Bassel, G. W. (2015) Digital single-cell analysis of plant organ development using 3DCellAtlas. Plant Cell, 27, 1018-1033.

Mooney, S. J., Pridmore, T. P., Helliwell, J. \& Bennett, M. J. (2012) Developing X-ray Computed Tomography to non-invasively image 3-D root systems architecture in soil. Plant Soil, 352, 1-22.

Nelson, C. J., Duckney, P., Hawkins, T. J., Deeks, M. J., Laissue, P. P., Hussey, P. J. \& Obara, B. (2015) Blobs and curves: object-based colocalisation for plant cells. Functional Plant Biology, 42, 471-485.

Obara, B., Fricker, M., Gavaghan, D. \& Grau, V. (2012a) Contrast-independent curvilinear structure detection in biomedical images. IEEE Trans. Image Process, 21, 2572-2581.

Obara, B., Grau, V. \& Fricker, M. D. (2012b) A bioimage informatics approach to automatically extract complex fungal networks. Bioinformatics, 28, 2374-2381.

Paredez, A. R., Somerville, C. R. \& Ehrhardt, D. W. (2006) Visualization of cellulose synthase demonstrates functional association with microtubules. Science, 312, 1491-1495.

Perez, A. J., Seyedhosseini, M., Deerinck, T. J., Bushong, E. A., Panda, S., Tasdizen, T. \& Ellisman, M. H. (2014) A workflow for the automatic segmentation of organelles in electron microscopy image stacks. Frontiers in Neuroanatomy, 8.

Pound, M. P., French, A. P., Wells, D. M., Bennett, M. J. \& Pridmore, T. P. (2012) CellSeT: Novel Software to Extract and Analyze Structured Networks of Plant Cells from Confocal Images. Plant Cell, 24, 1353-1361.

Rellán-Álvarez, R., Lobet, G., Lindner, H., Pradier, P.-L., Sebastian, J., Yee, M.-C., Geng, Y., Trontin, C., LaRue, T., Schrager-Lavelle, A., Haney, C. H., Nieu, R., Maloof, J., Vogel, J. P. \& Dinneny, J. R. (2015) GLO-Roots: an imaging platform enabling multidimensional characterization of soil-grown root systems. eLife. 10.7554/eLife.07597

Rousseau, D., Chéné, Y., Belin, E., Semaan, G., Trigui, G., Boudehri, K., Franconi, F. \& Chapeau-Blondeau, F. (2015) Multiscale imaging of plants: current approaches and challenges. Plant Methods, 11, 6.

Saar BG, Zeng Y, Freudiger CW, Liu YS, Himmel ME, Xie XS, Ding SY. (2010) Label-free, real-time monitoring of biomass processing with stimulated Raman scattering microscopy Angew Chem Int Ed Engl. 49,5476-9. 
Sampathkumar, A., Gutierrez, R., McFarlane, H. E., Bringmann, M., Lindeboom, J., Emons, A. M., Samuels, L., Ketelaar, T., Ehrhardt, D. W. \& Persson, S. (2013) Patterning and Lifetime of Plasma Membrane-Localized Cellulose Synthase Is Dependent on Actin Organization in Arabidopsis Interphase Cells. Plant Physiol., 162, 675-688.

Sampathkumar, A., Krupinski, P., Wightman, R., Milani, P., Berquand, A., Boudaoud, A., Hamant, O., Jönsson, H. \& Meyerowitz, E. M. (2014a) Subcellular and supracellular mechanical stress prescribes cytoskeleton behavior in Arabidopsis cotyledon pavement cells. eLife, 3.

Sampathkumar, A., Yan, A., Krupinski, P. \& Meyerowitz, Elliot, M. (2014b) Physical Forces Regulate Plant Development and Morphogenesis. Curr. Biol., 24, R475-R483.Schindelin, J., Arganda-Carreras, I., Frise, E., Kaynig, V., Longair, M., Pietzsch, T., Preibisch, S., Rueden, C., Saalfeld, S., Schmid, B., Tinevez, J.-Y., White, D. J., Hartenstein, V., Eliceiri, K., Tomancak, P. \& Cardona, A. (2012) Fiji: an open-source platform for biological-image analysis. Nat Meth, 9, 676-682.

Schleifenbaum, F., Elgass, K., Sackrow, M., Caesar, K., Berendzen, K., Meixner, A. J. \& Harter, K. (2010) Fluorescence intensity decay shape analysis microscopy (FIDSAM) for quantitative and sensitive live-cell imaging: a novel technique for fluorescence microscopy of endogenously expressed fusionproteins. Mol. Plant, 3, 555-562.

Schmidt, T., Pasternak, T., Liu, K., Blein, T., Aubry-Hivet, D., Dovzhenko, A., Duerr, J., Teale, W., Ditengou, F. A., Burkhardt, H., Ronneberger, O. \& Palme, K. (2014) The iRoCS Toolbox - 3D analysis of the plant root apical meristem at cellular resolution. Plant J., 77, 806-814.

Schneider, C. A., Rasband, W. S. \& Eliceiri, K. W. (2012) NIH Image to ImageJ: 25 years of image analysis. Nat Meth, 9, 671-675.

Schwarzländer, M., Dick, T. P., Meyer, A. J. \& Morgan, B. (2015) Dissecting redox biology using fluorescent protein sensors. Antioxidants \& Redox Signaling. DOI: 10.1089/ars.2015.6266

Schwarzlander, M., Fricker, M. D., Muller, C., Marty, L., Brach, T., Novak, J., Sweetlove, L. J., Hell, R. \& Meyer, A. J. (2008) Confocal imaging of glutathione redox potential in living plant cells. J. Microsc., 231, 299-316.

Schwarzländer, M., Logan, D. C., Johnston, I. G., Jones, N. S., Meyer, A. J., Fricker, M. D., \& Sweetlove, L. J. (2012). Pulsing of membrane potential in individual mitochondria: a stress-induced mechanism to regulate respiratory bioenergetics in arabidopsis. Plant Cell,. 24: 1188-1201,

Schwarzländer M., Wagner S., Ermakova Y.G., Belousov V.V., Radi R., Beckman J.S., Buettner G.R., Demaurex N., Duchen M.R., Forman H.J., Fricker M.D., Gems D., Halestrap A.P., Halliwell B., Jakob U., Johnston I.G., Jones N.S., Logan D.C., Morgan B., Müller F.L., Nicholls D.G., Remington S.J., Schumacker P.T., Winterbourn C.C., Sweetlove L.J., Meyer A.J., Dick T.P., and Murphy M.P.(2014). The "mitoflash" probe cpYFP does not respond to superoxide. Nature, 514 (7523), E12-4.

Serrano-Mislata, A., Schiessl, K. \& Sablowski, R. (2015) Active control of cell size generates spatial detail during plant organogenesis. Curr. Biol., 25, 29912996.

Shaw, S. L. \& Ehrhardt, D. W. (2013) Smaller, faster, brighter: advances in optical imaging of living plant cells. Ann. Rev. Plant Biol., 64, 351-375. 
Skibbe, H., Reisert, M., Schmidt, T., Brox, T., Ronneberger, O. \& Burkhardt, H. (2012) Fast rotation invariant 3d feature computation utilizing efficient local neighborhood operators. IEEE Trans. Pattern Analysis Machine Intelligence, , 34, 1563-1575.

Slovak, R., Göschl, C., Su, X., Shimotani, K., Shiina, T. \& Busch, W. (2014) A scalable open-source pipeline for large-scale root phenotyping of arabidopsis. Plant Cell, 26, 2390-2403.

Smal, I. \& Meijering, E. (2015) Quantitative comparison of multiframe data association techniques for particle tracking in time-lapse fluorescence microscopy. Medical Image Analysis, 24, 163-189.

Smith, S. \& Brady, J. M. (1997) SUSAN - a new approach to low level image processing. International Journal of Computer Vision, 23, 45-78.

Sozzani, R., Busch, W., Spalding, E. P. \& Benfey, P. N. (2014) Advanced imaging techniques for the study of plant growth and development. Trends Plant Sci., 19, 304-310.

Swanson, S. J., Choi, W. G., Chanoca, A. \& Gilroy, S. (2011) In vivo imaging of $\mathrm{Ca}^{2+}$, $\mathrm{pH}$, and reactive oxygen species using fluorescent probes in plants. Ann. Rev. Plant Biol. 62, 273-297.

Sun J., Miwa H., Downie J.A., and Oldroyd G.E.D. (2007). Mastoparan activates calcium spiking analogous to Nod factor-induced responses in Medicago truncatula root hair cells. Plant Physiol. 144, 695-702.

Waadt R, Hitomi K, Nishimura N, Hitomi C, Adams SR, Getzoff ED, Schroeder JI. 2014. FRET-based reporters for the direct visualization of abscisic acid concentration changes and distribution in Arabidopsis. eLife 3:e01739. doi: 10.7554/eLife.01739

Weissflog, I., Vogler, N., Akimov, D., Dellith, A., Schachtschabel, D., Svatos, A., et al. (2010). Toward in vivo chemical imaging of epicuticular waxes. Plant Physiol., 154, 604-610.

Yang, Z., Downie, H., Rozbicki, E., Dupuy, L. X. \& MacDonald, M. P. (2013) Light Sheet Tomography (LST) for in situ imaging of plant roots. Optics Express, 21, $16239-16247$.

Y Zeng, B G Saar, M G Friedrich, F Chen, and Y S Liu Imaging lignin-downregulated alfalfa using coherent anti-Stokes Raman scattering microscopy (2010) BioEnergy Research 3, 3.

Zhan, M., Crane, M. M., Entchev, E. V., Caballero, A., de Abreu, D. A. F., Ch'ng, Q. \& Lu, H. (2015) Automated Processing of Imaging Data through Multi-tiered Classification of Biological Structures Illustrated Using Caenorhabditis elegans. PLoS computational biology 11, e1004194.

Zumbusch A., Holtom, G.R. and Xie X.S., (1999) Three-dimensional vibrational imaging by coherent anti-stokes scattering Phys. Rev. Lett. 82, 4142.

\section{Figure Legends}

Figure 1. Shared methods of feature detection can be applied to diverse sources of data. (A) Panel A of figure 1, scheme XI from Micrographia (Hooke 1665) showing a transverse section of cork tissue. (B) Segmentation of the engraving using a watershed algorithm and Gaussian filter pre-processing. (C) CARS imaging of freshly transverse-sectioned cork tissue. Images were acquired using pump and Stokes wavelength of $797 \mathrm{~nm}$ and $1031 \mathrm{~nm}$ to excite the $\mathrm{CH}_{2}$ stretch at $2845 \mathrm{~cm}^{-1}$. 
(D) Segmentation of CARS data using identical filter and algorithm combination as (B).

Figure 2. Application of bioimaging informatics software for different scales of plant growth dynamics. Bars show the span of each package. Blue bars show software packages that are either openly available for download or are available within the academic domain upon request. Red bars represent commercial software packages.

\section{Supplemental Methods}

\section{CARS Microscopy}

The dual-wavelength picosecond excitation required for CARS microscopy was generated using an optical parametric oscillator (OPO) (Levante Emerald, APE, Berlin) pumped with a frequency doubled MOPA fibre laser (Emerald Engine, APE, Berlin). The pump laser generated a $1 \mathrm{ps}, 76 \mathrm{MHz}$ pulse train at $515.5 \mathrm{~nm}$. The OPO produced collinear signal and idler beams with perfect temporal overlap and provided continuous tuning over a range of wavelengths. The signal beam was used as the pump, ranging from 670 to $980 \mathrm{~nm}$ and the fundamental from the fibre laser $(1031 \mathrm{~nm})$ was used as the Stokes beam. The average power at the sample was between $15 \mathrm{~mW}$ and $30 \mathrm{~mW}$.

Imaging was performed using a modified inverted microscope and confocal laser scanner (IX71 and FV300, Olympus UK). A detailed description of the set, as applied to plant samples can be found in Mansfield et al., (2013) and Littlejohn et al., (2014). To maximise IR transmission the standard galvanometer scanning mirrors were replaced with silver galvanometric mirrors, the tube lens was replaced with a MgF2 coated lens and the confocal dichroic was replaced by a silver mirror with high reflectivity throughout the visible and NIR (21010, Chroma Technologies, USA). All imaging was performed using a 60X, 1.2 NA water immersion objective (UPlanS Apo, Olympus UK).

The CARS signal was detected in the epi-direction using the objective lens and separated from the pump and Stokes beams by a long-wave pass dichroic mirror (z850rdc-xr, Chroma Technologies, USA) and directed onto a photomultiplier tube (Hamamatsu R3896) at the rear microscope port. The anti-Stokes signal was isolated at each photodetector by a single band-pass filter centred at $750 \mathrm{~nm}$ (HQ750/210, Chroma Technologies USA). Images of cork were acquired using pump and Stokes wavelength of $797 \mathrm{~nm}$ and $1031 \mathrm{~nm}$ to excite the $\mathrm{CH}_{2}$ stretch at 2845 $\mathrm{cm}^{-1}$.

Watershed Analysis

The watershed anaysis of figure 1 was performed using ImageJ and Graylevel Watershed plugin Biomedical Imaging Group (BIG), EPFL Lausanne, Switzerland (http://bigwww.epfl.ch/sage/soft/watershed/). An 8-bit RGB digitised image of cork from Micrographia was sourced from an online repository (http://www.rarebookroom.org). The image was converted to 8-bit grayscale and a Gaussian filter with a sigma value of 2 pixels was applied. The 16-bit raw CARS data was also converted to 8-bit grayscale and a Gaussian filter of 6 pixel sigma was 
applied. Images were segmented with plugin settings of "Dark object / Bright background" with "8-connected" depth. A mask was added to the Micrographia segmentation to isolate the tissue from the background texture.

\section{Box / Appendix}

\section{Coherent Raman Scattering microscopy - its advantages and theory}

Fluorescence microscopy's reliance on fluorescent labelling is not compatible with low molecular weight biomolecules since it alters biochemistry. Alternative techniques based on vibrational spectroscopy provide in situ chemical analysis based on the vibrational frequencies of molecular bonds within a sample. Infrared absorption techniques have limited value for bio-imaging due to water absorption and the intrinsically low spatial resolution resulting from IR excitation, however Raman scattering provides analysis of IR vibrational frequencies with biocompatible excitation wavelengths. The drawback is that Raman scattering is an extremely weak effect with typical photon conversion efficiencies in biological materials being of the order of 1 in $10^{18}$. This severely limits Raman imaging of living systems since long acquisition times (100 ms to $1 \mathrm{~s}$ per pixel) at relatively high excitation powers (several hundred $\mathrm{mW}$ ) are required to detect most biomolecules with sufficient sensitivity. In plant tissues the lack of sensitivity is compounded by interference from autofluorescent pigments that overwhelm the Raman emission and virtually prohibits its application in all but a few areas of plant biology.

Far stronger Raman signals can be obtained using coherent Raman scattering (CRS), which achieves a signal enhancement via coherent excitation of a specific Raman mode of molecules under investigation (Maker and Terhune,1965). Pump and Stokes beams, with frequencies $\omega_{p}$ and $\omega_{S}$ respectively, are incident upon the sample with their frequency difference $\left(\omega_{p}-\omega_{s}\right)$ matched to the vibrational frequency of interest. Under this resonant condition, bonds are coherently driven by the beating excitation fields and a coherent Raman signal is produced. Due to the non-linear dependence of the signal on excitation intensity, further enhancement is achieved by excitation with ultra-short light pulses, which deliver high peak intensities at average powers comparable with those routinely used for fluorescence microscopy. Optimal excitation of CRS requires pulses with bandwidth corresponding to the line-width of the Raman band being investigated, which for biological molecules is typically of the order of $0.2 \mathrm{~nm}$ (corresponding to transform limited pulse width of a few picoseconds). When applied in a microscopy format CRS benefits from the non-linear nature of the process which confines the signal to a submicron focus that can be scanned in space, allowing 3D mapping of bio-molecules with sub-micron resolution (Zumbusch A, et al. 1999).

CRS was first applied to bio-imaging in 1999 and has now emerged as a powerful microscopy tool that has been applied with great effect to a wide range of biomedical applications (for review see Min et al., 2011) and can be combined with classifier schemes to automatically segment sub-cellular organelles with no external labelling 
(El-Mashtoly et al., 2014). Unfortunately CRS is severely limited in heavily pigmented samples, such as plant tissues. Therefore, despite its great potential to impact in plant biology, there have been very few examples of its application in this area and cases where it has been used rely on dried or processed plant materials (Weisflog et al. 2010, Zeng Y, et al. 2010, Saar et al., 2010).

CRS microscopy can be achieved by detecting either coherent anti-Stokes Raman scattering (CARS) or stimulated Raman scattering (SRS). CARS uses the antiStokes signal generated at frequency $\omega_{a s}=2 \omega_{p}-\omega_{s}$, which is spectrally isolated from the pump and Stokes beams. The anti-Stokes (blue) shift of the emission with respect to the excitation wavelengths makes CARS more resistant to autofluorescence than spontaneous Raman. However, for highly fluorescent samples such as plant tissues, the two-photon excited autofluorescence (also blueshifted with respect to the excitation) overwhelms the CARS signal Mansfield et al., (2013).

SRS relies on detecting changes in the intensities of the excitation fields that occur by virtue of stimulated excitation (Freudiger CW, et al. Science 322, 2008) When the difference frequency, $\omega_{p}-\omega_{s}$, matches a vibrational resonance the intensity of the Stokes beam, $I_{s}$, experiences a gain (SRG) while the intensity of the pump beam, Ip, experiences a loss (SRL). Modulating the intensity of either excitation beam modulates the SRS process and hence transfers an intensity modulation onto the other beam. The amplitude of the transferred intensity modulation is directly proportional to the concentration of target molecules and by modulating at frequencies above laser noise $(>1 \mathrm{MHz})$ can be detected with a lock-in amplifier with great sensitivity ( 1 in $10^{6}$ photons). Since SRS is detected at the same wavelength as the excitation fields it is not affected by fluorescent emission. However, as recently shown by Mansfield et al, SRS does succumb to interference from absorbing compounds. Single- or two- photon electronic absorption of either the pump or Stokes beams, despite reducing their intensity, does not produce a modulation transfer between the beams since there is no temporal correlation between the absorption of the different wavelengths. Modulation transfer does however occur for two-photon absorption (TPA) of the combined pump and Stokes beams since this process can only occur when both beams are incident upon the sample. In most samples this is an extremely weak effect, however in plant tissues the optical absorption is sufficiently strong across a broad spectral range and TPA overwhelms the SRS signal.

A major step forwards for Raman imaging in- $\square$ planta, recently published by Mansfield et al, was $\square$ to exploit the phase difference between $\square$ stimulated Raman gain (SRG), detected as a $\square$ gain in the Stokes beam and TPA, which results in a loss in the pump beam, to separate the two signals using phase-sensitive lock-in detection. Using this technique, we demonstrated the first application of in-situ spectroscopic Raman imaging in living pigmented plant tissues. Moreover, we have shown that with this technique it is possible to image the ingress of (unlabelled) low MW compounds in planta at the cellular level (Littlejohn et al. 2014). 

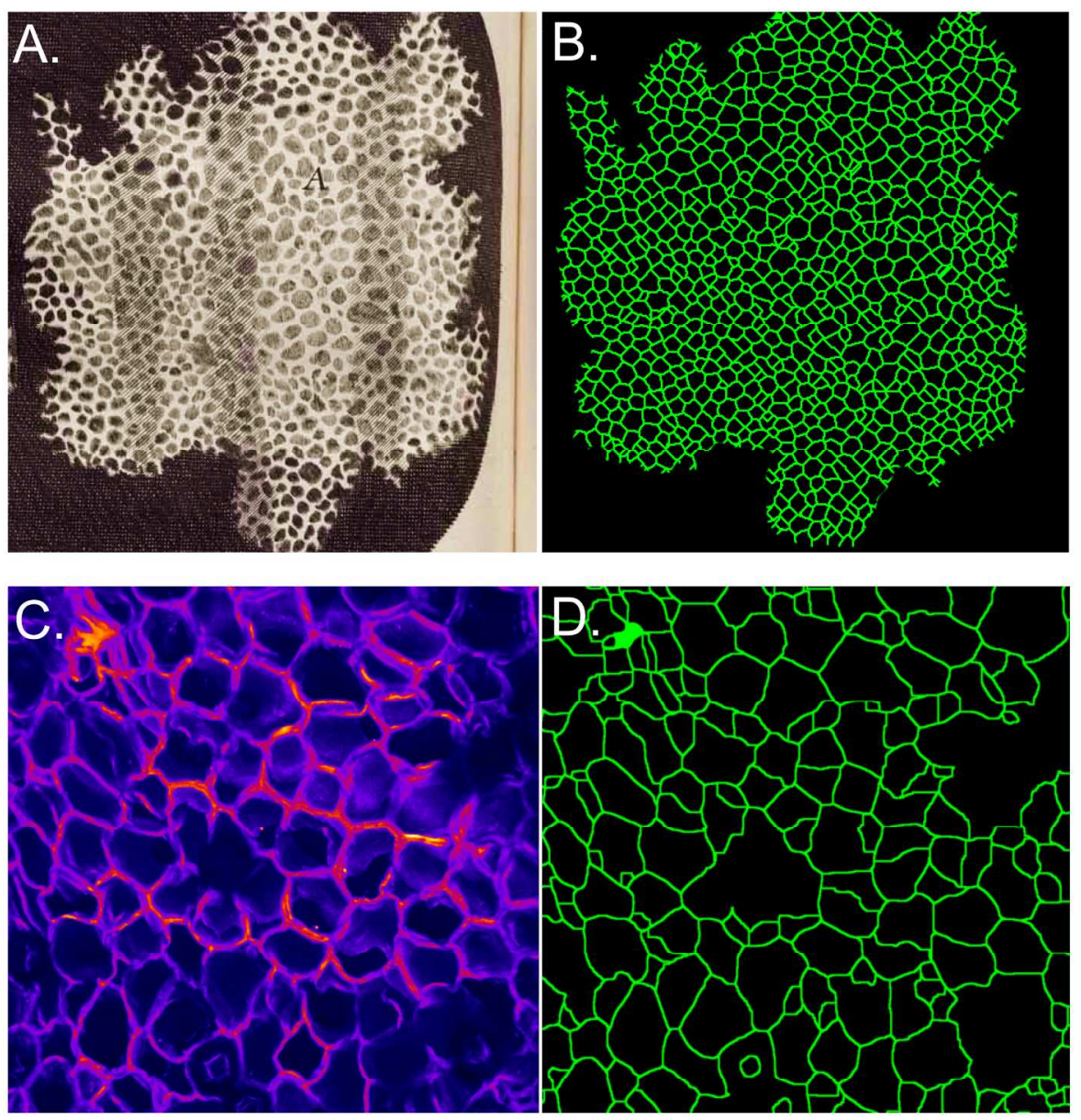

Figure 1. Shared methods of feature detection can be applied to diverse sources of data. (A) Panel A of figure 1, scheme XI from Micrographia (Hooke 1665) showing a transverse section of cork tissue. (B) Segmentation of the engraving using a watershed algorithm and Gaussian filter pre-processing. (C) CARS imaging of freshly transverse-sectioned cork tissue. Images were acquired using pump and Stokes wavelength of $797 \mathrm{~nm}$ and $1031 \mathrm{~nm}$ to excite the $\mathrm{CH} 2$ stretch at $2845 \mathrm{~cm}-1$. (D) Segmentation of CARS data using identical filter and algorithm combination as (B). $287 \times 302 \mathrm{~mm}(200 \times 200$ DPI $)$ 


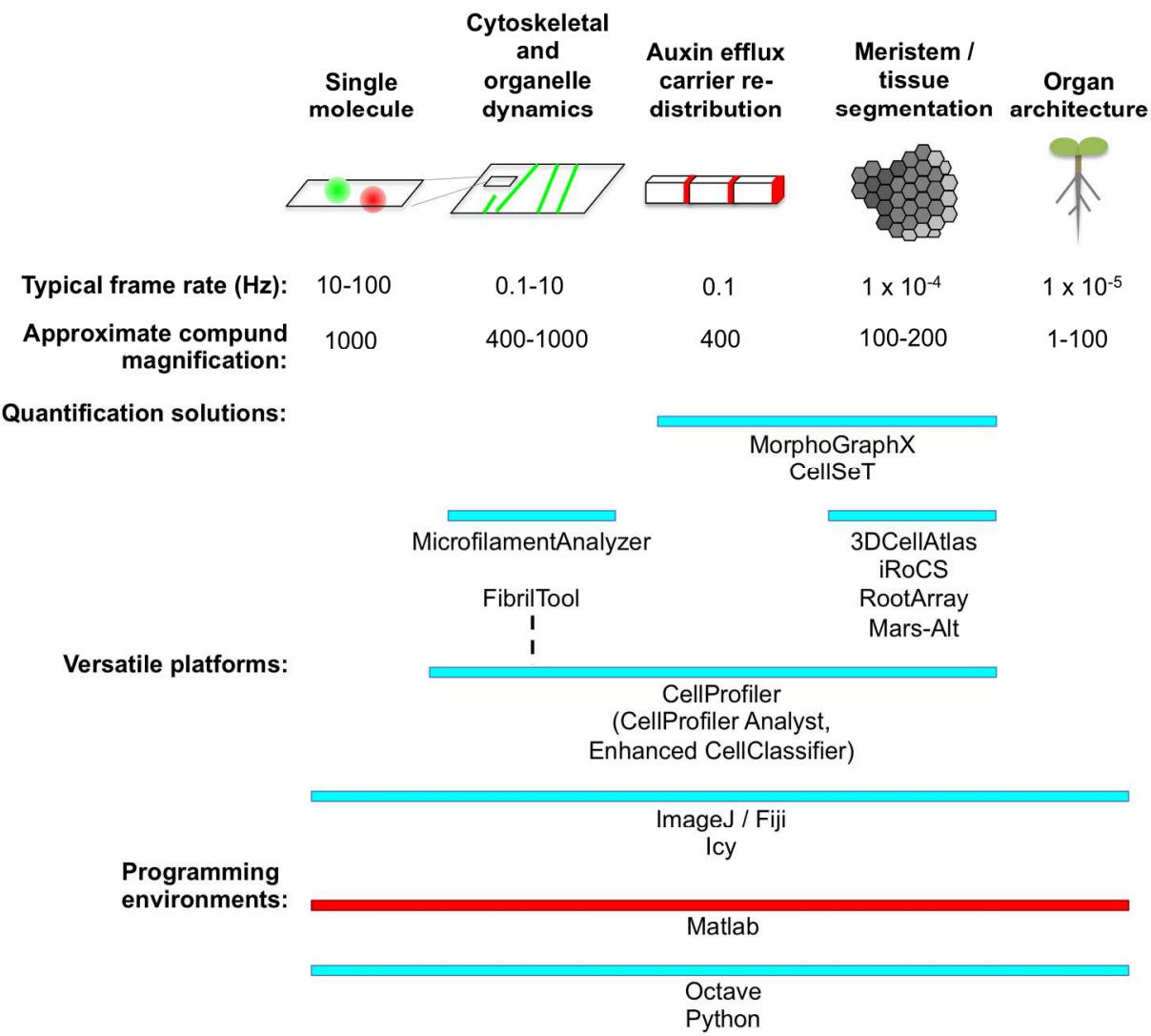

Figure 2. Application of bioimaging informatics software for different scales of plant growth dynamics. Bars show the span of each package. Blue bars show software packages that are either openly available for download or are available within the academic domain upon request. Red bars represent commercial software packages.

$749 \times 648 \mathrm{~mm}(72 \times 72 \mathrm{DPI})$ 
Cell theory has officially reached 350 years of age as the first use of the word 'cell' in a biological context can be traced to a description of plant material by Robert Hooke in his historic publication "Micrographia: or some physiological definitions of minute bodies". The 2015 Royal Microscopical Society Botanical Microscopy meeting was a celebration of the streams of investigation initiated by Hooke to understand at the sub-cellular scale how plant cell function and form arises. Hooke identified the polar-nature of plant cells by noting that their appearance was different depending on the angle at which the cork was cut. Each cell has a controlled shape that interlocks with its neighbours. How does this occur? Much of the work presented during the meeting described advances made in understanding how the dynamics of cellular components contribute to this process. The Honorary Fellowships awarded to Professors Chris Hawes, Peter Hepler and David Ehrhardt helped highlight the importance of quantitative microscopy; the measurement of feature and behaviours in micrographs. Over the past decade there have been significant advances in the application of computer algorithms to extract quantities from images and these have had a large impact upon botanical microscopy. The field of computer science has developed methods to recognise and track objects that have biological meaning. Botanists have benefitted enormously from cross-disciplinary interactions with computer scientists and statisticians to develop fully-formed software packages that are tailored for plant science. Critically, this software can (for the most part) be used on desktop personal computers. Moreover the majority of software is available free of charge to the academic and general public alike. In this review we provide a guide for the uninitiated to find powerful but easy-to-use software solutions for botanical light microscopy. This is accompanied by an overview of the principles driving the object-based imaging revolution and more technical descriptions of the algorithms at the heart of some of these packages. We also discuss the oft-neglected issue of measuring the performance of algorithms and the exciting possibilities offered by biosensors and label-free imaging. 